\title{
Increased expression of immediate early response gene 3 protein promotes aggressive progression and predicts poor prognosis in human bladder cancer
}

Jianheng Ye $\mathrm{e}^{1,2+}$, Yanqiong Zhang ${ }^{2,3 \dagger}$, Zhiduan Cai ${ }^{4}$, Minyao Jiang ${ }^{1}$, Bowei Li ${ }^{1}$, Guo Chen ${ }^{1}$, Yanru Zeng ${ }^{1}$, Yuxiang Liang ${ }^{1}$, Shulin $\mathrm{Wu}^{2}$, Zongwei Wang ${ }^{2}$, Huichan $\mathrm{He}^{1,6^{*}}$, Weide Zhong ${ }^{1,5^{*}}$ and Chin-Lee Wu ${ }^{2^{*}}$

\begin{abstract}
Background: Immediate early response gene 3 (IER3) is a stress-inducible gene, which exerts diverse effects in regulating cell apoptosis and cell cycle. Growing evidence shows that IER3 functions either as an oncogene or a tumor suppressor in various human cancers with a cancer type-dependent manner. However, the involvement of IER3 in human bladder cancer (BCa) has not been elucidated. In the current study, we aimed to investigate the expression pattern and the clinical significance of IER3 in BCa.
\end{abstract}

Methods: We performed immunohistochemistry analysis to examine the subcellular localization and the expression levels of IER3 protein in 88 BCa specimens obtained from Department of Pathology in Massachusetts General Hospital. The associations of IER3 protein expression with various clinicopathological features and patients' overall survival were statistically evaluated.

Results: IER3 protein was mainly expressed in the cytoplasm in bladder cancer cell. Of 88 BCa tissue specimens, 39 (44.3\%) showed high expression of IER3 protein and 49 (55.7\%) showed low expression. High IER3 protein expression was significantly associated with high pathologic nodal stage $(p=0.018)$. Kaplan-Meier analysis revealed that the overall survival of BCa patients with overexpression of IER3 protein was shorter than that with low expression $(p<0.01)$. Multivariate analysis by Cox regression further identified IER3 as an independent prognostic factor of BCa patients $(p=0.010)$.

Conclusions: Our findings suggest for the first time that the increased expression of IER3 protein may promote the aggressive progression of BCa. Importantly, IER3 may be a potential prognostic marker for BCa patients.

Keywords: Immediate early response gene 3, Bladder cancer, Clinicopathological feature, Prognosis

\footnotetext{
* Correspondence: xiaohejian@21cn.com; zhongwd2009@live.cn; cwu2@mgh.harvard.edu

${ }^{\dagger}$ Jianheng Ye and Yanqiong Zhang contributed equally to this work.

'Department of Urology, Guangdong Key Laboratory of Clinical Molecular Medicine and Diagnostics, Guangzhou First People's Hospital, Guangzhou Medical University, Guangzhou 510180, China

${ }^{2}$ Departments of Urology and Pathology, Massachusetts General Hospital and Harvard Medical School, Boston, MA 02114, USA

Full list of author information is available at the end of the article
}

(c) The Author(s). 2018 Open Access This article is distributed under the terms of the Creative Commons Attribution 4.0 International License (http://creativecommons.org/licenses/by/4.0/), which permits unrestricted use, distribution, and reproduction in any medium, provided you give appropriate credit to the original author(s) and the source, provide a link to the Creative Commons license, and indicate if changes were made. The Creative Commons Public Domain Dedication waiver (http://creativecommons.org/publicdomain/zero/1.0/) applies to the data made available in this article, unless otherwise stated. 


\section{Background}

Bladder cancer (BCa) was the fourth most common cancer in men and the twelfth most common cancer in women in United States in 2016 [1]. Although there have been great advances in bladder carcinogenesis, the mortality rate of $\mathrm{BCa}$ is still high, due to its complex etiology and insufficient therapeutic strategies. A multivariate analysis showed that an increasing death rate of $\mathrm{BCa}$ was associated with multiple environmental exposures, such as smoking, air pollution, well water, urban residence and mining employment [2]. Most of $\mathrm{BCa}$ occur as non-muscle-invasive cancer, but there are still approximately $25 \%$ of them have muscle-invasive or metastatic disease, which leads to a poor outcome [3]. Therefore, it is of great clinical significance to discover novel and efficient molecular markers to develop more accurate diagnosis and prognosis methods for $\mathrm{BCa}$ patients.

Immediate early response gene 3 (IER3), also known as IEX-1, Dif-2, gly96 or p22/PRG-1, is a stress-inducible gene, which is rapidly regulated by multiple factors, including transcription factors, inflammatory cytokines, viral infection, chemical carcinogens, growth factors and hormones [4]. Under a wide range of stress, IER3 activation exerts diverse effects in regulating cell apoptosis and cell cycle with its distinct domains [5]. Accumulating studies have reported that IER3 may be associated with various signaling pathways, such as Nuclear factor kappa B (NF-kB) pathway and Mitogen-activated protein kinase (MAPK) /Extracellular regulated protein kinases (ERK) pathway, and may functions either as an oncogene or a tumor suppressor [6-8].

IER3 expression has been observed in a wide range of human epithelial tissues [9]. Growing evidence also shows that the aberrant expression of IER3 may be associated with prognosis in patients with multiple cancers [4]. However, the involvement of IER3 in BCa has not been elucidated. In this present study, we aimed to examine the expression pattern of IER3 protein in $\mathrm{BCa}$ tissues, and to evaluate its clinical significance in this malignancy.

\section{Methods}

\section{Patients and tissues}

This study used the same cohorts of BCa patients and tissue samples with our previous study [10]. All eighty-eight $\mathrm{BCa}$ tissue samples obtained from eighty-eight $\mathrm{BCa}$ patients who underwent cystectomy were collected from Massachusetts General Hospital between 2002 and 2010. The BCa patients' detail clinical information was shown in Table 1. All cases were re-reviewed by CLW and JHY (two authors) according to the newest version of World Health Organization classification of tumor of the bladder [11].
Table 1 Associations between IER3 protein expression and various clinicopathological characteristics of 88 BCa patients underwent cystectomy between 2002 and 2010

\begin{tabular}{llll}
\hline & IER3 (low) & IER3 (high) & $P$ \\
\hline Number of patients, no. \% & $49(55.7)$ & $39(44.3)$ & \\
Gender, no.\% & & & 0.799 \\
$\quad$ Female & $12(24.5)$ & $8(20.5)$ & \\
$\quad$ Male & $37(75.5)$ & $31(79.5)$ & \\
Age at Surgery, median(IQR) & $70(62-75)$ & $72(62-80)$ & 0.462 \\
pT, no. \% & & & 0.493 \\
$\quad<=$ pT2 & $16(32.7)$ & $10(25.6)$ & \\
> =pT3 & $33(67.3)$ & $29(74.4)$ & \\
pN, no. \% (n= 79) & & & 0.018 \\
pN(-) & $33(76.7)$ & $18(50.0)$ & \\
pN(+) & $10(23.3)$ & $18(50.0)$ & \\
LVI, no. \% & & & \\
$\quad$ LVI(-) & & & \\
LVI(+) & $31(63.3)$ & $20(51.3)$ & \\
PNI, no. \% & $18(36.7)$ & $19(48.7)$ & \\
PNI(-) & & & \\
PNI(+) & & & \\
STSM, no. \% & & & \\
margin(-) & $37(75.5)$ & $28(71.8)$ & \\
margin(+) & $12(24.5)$ & $11(28.2)$ & \\
Metastasis, no. \% & & & \\
Mets(-) & & & \\
Mets(+) & & & \\
\hline
\end{tabular}

Bold values indicate that they are less than 0.05

\section{Immunohistochemistry and Immunoreactive score}

Following the immunohistochemistry (IHC) protocol we described in our previous study [10], IER3 protein expression level was assessed by IHC using a polyclonal goat anti-IER3 antibody (Santa-Cruz biotechnology, CA). Detail information of IHC protocol was provided in Additional file 1. After performing IHC, the IER3 protein expression level in each tissue section was evaluated according to the immunostaining intensity and percentage. Immunoreactive score (IRS) was obtained by multiplying intensity and percentage of immunostaining. The intensity of immunostaining graded from 0 to 3: 0 (negative), 1 (weak), 2 (moderate), and 3 (strong); the percentage of staining cells were classified as 0-4: 0 (negative), 1 (<=10\%), 2 (11-50\%), 3 (51-80\%), and 4 (>80\%). The final IRS (ranged from 0 to 12 ) was determined: 0-3 as low IER3 protein expression, and 4-12 as high IER3 protein expression [12]. The IRS of IER3 immunostaining of each tissue section was scored by two investigators (CLW and JHY) independently in a blind manner without any knowledge of the patient clinical characteristics. 
The IRSs evaluated by two investigators respectively were compared. In order to reach a consensus, different IRSs of the same tissue section were reevaluated through a re-examination of the intensity of IER3 immunostaining by both investigators.

\section{Statistics}

All statistical analyses in this study were performed using Stata14 software (College Station, TX, USA).The associations between expression level of IER3 protein and $\mathrm{BCa}$ patients' clinicopathological data were analyzed by two statistical methods (Pearson's Chi-squared test or Fisher's exact test). Kaplan-Meier method was using to estimate the BCa patients' overall survival. Then, log-rank test was used for assessing differences. Cox proportional hazard regression models were used to perform univariate and multivariate survival analyses. Hazard ratios (HR) and the corresponding $95 \%$ confidence intervals (CI) were used to express the relative risks of dying. Two-sided with $p<0.05$ was considered as statistically significant.

\section{Results}

\section{Protein expression of IER3 in BCa}

IER3 protein's expression in $88 \mathrm{BCa}$ patients' tissue samples was identified by IHC. As shown in Fig. 1, IER3 protein was mainly localized in the cytoplasm of $\mathrm{BCa}$ cells. All specimens were divided into 2 groups: high IER3 protein expression group, containing 39 (44.3\%) BCa patients; low IER3 protein expression group, containing $49(55.7 \%)$ BCa patients. Statistical analysis showed that $\mathrm{BCa}$ patients with high expression of IER3 protein more frequently had high pathologic nodal stage $(\mathrm{pN})(p=0.018$, Table 1$)$. However, no significant associations were observed between IER3 protein expression level and other clinicopathological features, including patients' age, pathological stage ( $\mathrm{pT})$, gender, perineural invasion (PNI), lymphvascular invasion (LVI), soft tissue surgical margins (STSM) and distant metastasis.

\section{High IER3 protein expression was an independent indicator of poor prognosis in BCa patients}

After analyzed by Kaplan-Meier method, the curves showed that $\mathrm{BCa}$ patients in high IER3 protein expression group had worse overall survival compared with those in low IER3 protein expression group ( $p=0.002$,Fig. 2). Additionally, the univariate analysis demonstrated that old patients' age $(p=0.029)$, high $\mathrm{pT}(p=0.006)$, high $\mathrm{pN}$ $(p<0.001)$, positive LVI $(p=0.006)$, positive STSM $(p=$ $0.002)$, positive distant metastasis $(p=0.001)$ and high expression of IER3 protein $(p=0.003)$ were all associated with poor survival of BCa patients significantly (Table 2). The multivariate survival comparison indicated that patients' age $(p=0.012), \mathrm{pN}(p=0.007)$ and IER3 protein expression $(p=0.010)$ can act as the independent prognostic indicators of $\mathrm{BCa}$ (Table 2).

\section{Discussion}

IER3 was initially identified as a radiation-inducible protein in squamous carcinomas [13]. It has been demonstrated to response to quite distinct stress or cellular stimuli immediately. Among these stimulating factors, NF-кB, p53, SP1 and AP1 are well-known to be involved into tumor development [6]. Increasing evidence show that IER3 may play complex roles in cell apoptosis in different manners [4-6]. In a mitochondria-dependent environment, IER3 up-regulated can reduce the production of intracellular reactive oxygen species by facilitating the degradation of the inhibitor of F1 catalytic sector, which can prevent apoptosis at its initial phase [14]. On the other hand, IER3 was identified to mediate the NF- $\mathrm{kB}$ signaling pathway by interacting with RelA/p65 subunit. This modification can contribute to inducing the expression of
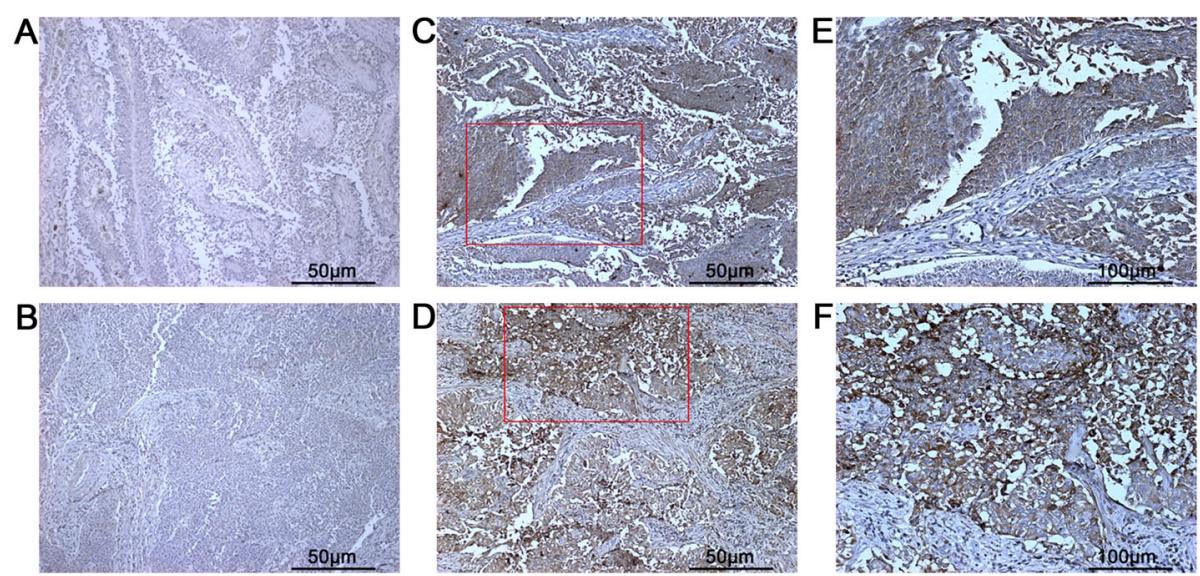

Fig. 1 Representative images of IER3 immunostaining in BCa tissue specimens. Negative ( $\mathbf{a}$ and $\mathbf{b}$ ) and positive (c and $\mathbf{d}$ ) expression of IER3 protein are shown (Magnification: 100X). Magnified images (e and $\mathbf{f}$ ) of two immunostaining regions marked by red boxes in $\mathbf{c}$ and $\mathbf{d}$ (Magnification: 200X) 


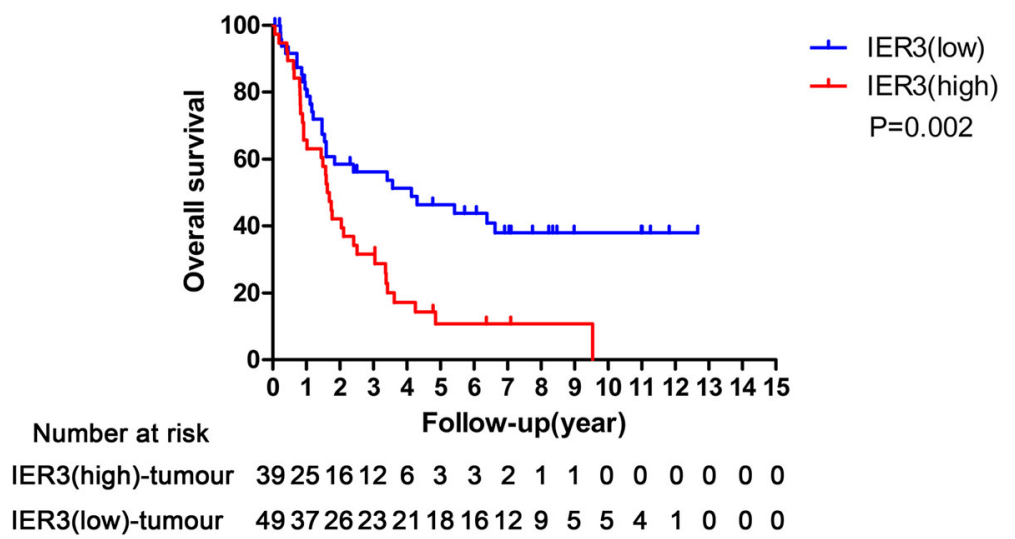

Fig. 2 Kaplan-Meier curves representing the overall survival of 88 patients treated with cystectomy for bladder cancer stratified by IER3 status ( $p=0.002)$

anti-apoptotic NF- $\mathrm{kB}$ target genes and then supporting cell apoptosis [15]. Besides the impact of IER3 on NF-kB signaling pathway, some studies indicated the IER3 might act as a regulator of ERK1/2 pathway. A phenomenon found by Letourneux et al. [16] showed that ERK activation led to IER3 phosphorylation. Simultaneously, the p-IER3 can enhance ERK phosphorylation by preventing its dephosphorylation factor B56-containing PP2A. The roles of IER3 sustaining ERK1/2 phosphorylation and promoting the tumor development were found in pancreatic cancer [17], lung adenocarcinoma [18] and Hodgkin lymphoma [19]. Moreover, IER3 were found to play crucial roles in regulating tumor growth. Han et al. [20] analyzed the clinical significance of IER3 expression in 77 ovarian carcinoma patients by using immunohistochemistry, and found a decreased expression of IER3 in ovarian carcinoma tissues compared to cystadenoma and borderline tumors tissues. They also indicated that the decreased expression of IER3 was associated with a short survival time of patients with this cancer. They subsequently showed a positive correlation between IER3 expression and anti-apoptotic activity of ovarian cancer cell. In breast cancer, estrogen was reported to effectively up-regulated the expression of IER3 [21]. Furthermore, Yang et al. [22]

Table 2 Prognostic value of IER3 protein expression for the overall survival by Cox proportional hazards model

\begin{tabular}{|c|c|c|c|c|c|c|}
\hline & \multicolumn{3}{|l|}{ Univariable } & \multicolumn{3}{|l|}{ Multivariable } \\
\hline & Hazard Ratio & $95 \% \mathrm{Cl}$ & $P$ & Hazard Ratio Ratio & $95 \% \mathrm{Cl}$ & $p$ \\
\hline \multicolumn{7}{|l|}{ Gender } \\
\hline Male vs. Female & 1.14 & $0.62-2.10$ & 0.681 & & & \\
\hline Age & 1.03 & $1.00-1.05$ & 0.029 & 1.04 & $1.01-1.07$ & 0.012 \\
\hline \multicolumn{7}{|l|}{ pT } \\
\hline$>=$ pT3 vs. $<=$ pT2 & 2.39 & $1.29-4.42$ & 0.006 & 1.54 & $0.71-3.32$ & 0.272 \\
\hline \multicolumn{7}{|l|}{$\mathrm{pN}$} \\
\hline $\mathrm{pN}(+)$ vs. $\mathrm{pN}(-)$ & 3.41 & $1.95-5.99$ & $<0.001$ & 2.35 & $1.26-4.38$ & 0.007 \\
\hline \multicolumn{7}{|l|}{ LVI } \\
\hline $\operatorname{LVI}(+)$ Vs. LVI(-) & 2.03 & $1.22-3.37$ & 0.006 & 0.99 & $0.52-1.89$ & 0.987 \\
\hline \multicolumn{7}{|l|}{ PNI } \\
\hline $\mathrm{PNI}(+)$ vs. PNI(-) & 1.03 & $0.56-1.87$ & 0.935 & & & \\
\hline \multicolumn{7}{|l|}{ STSM } \\
\hline STSM(+) vs. STSM(-) & 2.62 & $1.41-4.88$ & 0.002 & 2.02 & $0.96-4.27$ & 0.065 \\
\hline \multicolumn{7}{|l|}{ Metastasis } \\
\hline Mets(+) vs. Mets(-) & 2.35 & $1.41-3.97$ & 0.001 & 1.81 & $0.98-3.33$ & 0.058 \\
\hline \multicolumn{7}{|l|}{ IER3 IHC } \\
\hline IER3(high) vs. IER3(low) & 2.16 & $1.29-3.13$ & 0.003 & 2.21 & $1.21-4.04$ & 0.010 \\
\hline
\end{tabular}

Bold values indicate that they are less than 0.05 
showed IER3 was overexpressed in invasive breast cancer tissues compared with preinvasive cancer tissues. In vitro experimental system, IER3 was found to reduce the apoptotic activity of T47D and MCF-7 cells. Akilov et al. [23] revealed that IER3 up-regulation in Sézary cells might result in decreased expression levels of reactive oxygen species; as a downstream target of TNF- $\alpha$-induced pathway, IER3 could protect Sézary cells from TNF- $\alpha$-induced apoptosis. Sasada et al. [24] found that IER3 protein showed high expression in 41 patients and low expression in 37 patients with pancreatic cancer; statistically, patients with IER3 overexpression had a significantly better survival than those with low expression. In colon cancer, Nambiar et al. [25] built two different mouse models with hyperplasic or dysplastic preneoplastic aberrant crypt foci (ACF). The gene expression analysis based on ACF lesions showed that IER3 expression was increased in low risk ACF mice compared to high risk ACF mice; further immunohistochemistry analysis showed the positive staining of IER3 in adjacent normal-appearing colonic while the negative staining in the tumor crypts from the same patients. These findings suggest that the aberrant expression of IER3 may be implicated into carcinogenesis, progression and patients' outcome of various human cancers.

In present study, we determined that IER3 protein was overexpressed in the cytoplasm of cancer cells in BCa tissues specimens and that high IER3 protein expression was distinctly associated with high pathologic nodal stage. Notably, high IER3 protein expression also predicted poor overall survival in BCa patients.

\section{Conclusions}

Our findings suggest for the first time that the increased expression of IER3 protein may promote the aggressive progression of $\mathrm{BCa}$. Importantly, IER3 may be a potential prognostic marker for $\mathrm{BCa}$ patients. Further investigations on molecular mechanisms underlying IER3 involvement in $\mathrm{BCa}$ are required.

\section{Additional file}

Additional file 1: Immunohistochemistry Protocol. (DOCX $23 \mathrm{~kb}$ )

\footnotetext{
Abbreviations

ACF: Aberrant crypt foci; AP-1: Activator protein 1; BCa: Bladder cancer; Cl: Confidence intervals; ERK: Extracellular regulated protein kinases; HR: Hazard ratios; IER3: Immediate early response gene 3; IHC: Immunohistochemistry; IQR: Interquartile Range; IRS: Immunoreactive score; LVl: Lymphvascular invasion; MAPK: Mitogen-activated protein kinase; Mets: Metastasis; NF-kB: Nuclear factor kappa B; P: p-value; pN: Pathologic nodal stage; PNI: Perineural invasion; PT: Pathological stage; SP1: Specificity protein 1; STSM: Soft tissue surgical margins; TNFa: Tumor necrosis factor alpha

Funding

This work was supported by grants from National Key Basic Research Program of China (2015CB553706), National Natural Science Foundation of
}

China $(81571427,81470983,81641102)$, Science and Technology Project of Guangdong Province (2016A020215018, 2013B021800055), Guangzhou Municipal Science and Technology Project (2014 J4100072), Projects of Guangdong Key Laboratory of Clinical Molecular Medicine and Diagnostics \& $\mathrm{NIH} / \mathrm{NCl}$ P01 CA120964. These foundations did not interfere in the design of the study and collection, analysis, and interpretation of data and in writing the manuscript.

\section{Availability of data and materials}

The datasets used and/or analysed during the current study are available from the corresponding author on reasonable request.

\section{Authors' contributions}

WDZ, CLW, HCH: participated in study design and coordination, analysis and interpretation of data, material support for obtained funding, and supervised study. JHY and YQZ: performed most of the experiments, drafted and revised the manuscript. SLW and ZWW: performed statistical analysis. ZDC, MYJ, BWL, GC, YRZ and YXL: carried out the experiments and samples collection. All authors have read and approved the final version of the manuscript.

\section{Ethics approval and consent to participate}

This study was approved by the Hospital Ethics Committee of Massachusetts General Hospital. All of the patients gave informed consents before undergoing surgery. Written informed consent was obtained from all patients before the study. The principles of the 1964 Helsinki Declaration were followed in this study. In order to protect patients' privacy, we used Case No. to replace patients' names.

\section{Consent for publication}

Not applicable.

\section{Competing interests}

The authors declare that they have no competing interests.

\section{Publisher's Note}

Springer Nature remains neutral with regard to jurisdictional claims in published maps and institutional affiliations.

\section{Author details}

'Department of Urology, Guangdong Key Laboratory of Clinical Molecular Medicine and Diagnostics, Guangzhou First People's Hospital, Guangzhou Medical University, Guangzhou 510180, China. ${ }^{2}$ Departments of Urology and Pathology, Massachusetts General Hospital and Harvard Medical School, Boston, MA 02114, USA. Institute of Chinese Materia Medica, China Academy of Chinese Medical Sciences, Beijing 100700, China. ${ }^{4}$ Southern Medical University, Guangzhou 510515, China. ${ }^{5}$ Department of Urology, Guangzhou First People's Hospital, Guangzhou Medical University, Guangzhou 510180, China. EUrology Key Laboratory of Guangdong Province, The First Affiliated Hospital of Guangzhou Medical University, Guangzhou Medical University, Guangzhou 510230, China.

Received: 13 March 2017 Accepted: 24 August 2018

Published online: 24 September 2018

\section{References}

1. Siegel RL, Miller KD, Jemal A. Cancer statistics, 2016. CA Cancer J Clin. 2016; 66(1):7-30.

2. Smith ND, Prasad SM, Patel AR, Weiner AB, Pariser JJ, Razmaria A, Maene C, Schuble T, Pierce B, Steinberg GD. Bladder Cancer mortality in the United States: a geographic and temporal analysis of socioeconomic and environmental factors. J Urol. 2016;195(2):290-6.

3. Kamat AM, Hahn NM, Efstathiou JA, Lerner SP, Malmström PU, Choi W, Guo CC, Lotan Y, Kassouf W. Bladder cancer. Lancet. 2016:388(10061):2796-810.

4. Wu MX, Ustyugova IV, Han L, Akilov OE. Immediate early response gene X-1, a potential prognostic biomarker in cancers. Expert Opin Ther Targets. 2013; 17(5):593-606.

5. Shen L, Guo J, Santos-Berrios C, Wu MX. Distinct domains for anti- and proapoptotic activities of IER3. J Biol Chem. 2006:281(22):15304-11.

6. Arlt $\mathrm{A}$, Schäfer $\mathrm{H}$. Role of the immediate early response 3 (IER3) gene in cellular stress response, inflammation and tumorigenesis. Eur J Cell Biol. 2011;90(6-7):545-52. 
7. Wu MX, Ao Z, Prasad KV, Wu R, Schlossman SF. IEX-1L, an apoptosis inhibitor involved in NF-kappaB-mediated cell survival. Science. 1998; 281(5379):998-1001.

8. de Laval B, Pawlikowska P, Barbieri D, Besnard-Guerin C, Cico A, Kumar R, Gaudry M, Baud V, Porteu F. Thrombopoietin promotes NHEJ DNA repair in hematopoietic stem cells through specific activation of Erk and NF-KB pathways and their target, IEX-1. Blood. 2014;123(4):509-19.

9. Feldmann KA, Pittelkow MR, Roche PC, Kumar R, Grande JP. Expression of an immediate early gene, IER3, in human tissues. Histochem Cell Biol. 2001; 115(6):489-97.

10. Wu S, Ye J, Wang Z, Lin SX, Lu M, Liang Y, Zhu X, Olumi AF, Zhong WD, Wu $\mathrm{CL}$. Expression of aromatase in tumor related stroma is associated with human bladder cancer progression. Cancer Biol Ther. 2018;19(3):175-80.

11. Humphrey PA, Moch H, Cubilla AL, Ulbright TM, Reuter VE. The 2016 WHO classification of Tumours of the urinary system and male genital organs-part B: prostate and bladder Tumours. Eur Urol. 2016;70(1):106-19.

12. Specht E, Kaemmerer D, Sänger J, Wirtz RM, Schulz S, Lupp A. Comparison of immunoreactive score, HER2/neu score and $\mathrm{H}$ score for the immunohistochemical evaluation of somatostatin receptors in bronchopulmonary neuroendocrine neoplasms. Histopathology. 2015; 67(3):368-77.

13. Kondratyev AD, Chung KN, Jung MO. Identification and characterization of a radiation-inducible glycosylated human early-response gene. Cancer Res. 1996;56(7):1498-502.

14. Shen L, Zhi L, Hu W, Wu MX. IEX-1 targets mitochondrial F1Fo-ATPase inhibitor for degradation. Cell Death Differ. 2009:16(4):603-12.

15. Arlt A, Rosenstiel P, Kruse ML, Grohmann F, Minkenberg J, Perkins ND, Fölsch UR, Schreiber S, Schäfer H. IEX-1 directly interferes with ReIA/p65 dependent transactivation and regulation of apoptosis. Biochim Biophys Acta. 2008;1783(5):941-52.

16. Letourneux C, Rocher G, Porteu F. B56-containing PP2A dephosphorylate ERK and their activity is controlled by the early gene IEX-1 and ERK. EMBO J. 2006:25(4):727-38.

17. Garcia MN, Grasso D, Lopez-Millan MB, Hamidi T, Loncle C, Tomasini R, Lomberk G, Porteu F, Urrutia R, lovanna JL. IER3 supports KRAS ${ }^{G 12 D}$ dependent pancreatic cancer development by sustainingERK1/2 phosphorylation. J Clin Invest. 2014;124(11):4709-22.

18. Ito T, Ozaki S, Chanasong R, Mizutani Y, Oyama T, Sakurai H, Matsumoto I, Takemura H, Kawahara E. Activation of ERK/ER3/PP2A-B56Y-positive feedback loop in lung adenocarcinoma by allelic deletion of B56Y gene. Oncol Rep. 2016;35(5):2635-42.

19. Locatelli SL, Careddu G, Stirparo GG, Castagna L, Santoro A, Carlo-Stella C. Dual PI3K/ERK inhibition induces necroptotic cell death of Hodgkin lymphoma cells through IER3 downregulation. Sci Rep. 2016;6:35745.

20. Han L, Geng L, Liu X, Shi H, He W, Wu MX. Clinical significance of IEXexpression in ovarian carcinoma. Ultrastruct Pathol. 2011;35(6):260-6.

21. Rasmussen LM, Frederiksen KS, Din N, Galsgaard E, Christensen L, Berchtold MW, Panina S. Prolactin and oestrogen synergistically regulate gene expression and proliferation of breast cancer cells. Endocr Relat Cancer. 2010;17(3):809-22.

22. Yang C, Trent S, Ionescu-Tiba V, Lan L, Shioda T, Sgroi D, Schmidt EV. Identification of cyclin D1- and estrogen- regulated genes contributing to breast carcinogenesis and progression. Cancer Res. 2006;66(24):11649-58.

23. Akilov OE, Wu MX, Ustyugova IV, Falo LD Jr, Geskin LJ. Resistance of Sézary cells to TNF-a-induced apoptosis is mediated in part by a loss of TNFR1 and a high level of the IER3 expression. Exp Dermatol. 2012;21(4):287-92.

24. Sasada T, Azuma K, Hirai T, Hashida H, Kanai M, Yanagawa T, Takabayashi A. Prognostic significance of the immediate early response gene X-1 (IER3) expression in pancreatic cancer. Ann Surg Oncol. 2008;15(2):609-17.

25. Nambiar PR, Nakanishi M, Gupta R, Cheung E, Firouzi A, Ma XJ, Flynn C, Dong M, Guda K, Levine J, Raja R, Achenie L, Rosenberg DW. Genetic signatures of high- and low-risk aberrant crypt foci in a mouse model of sporadic colon cancer. Cancer Res. 2004;64(18):6394-401.

Ready to submit your research? Choose BMC and benefit from:

- fast, convenient online submission

- thorough peer review by experienced researchers in your field

- rapid publication on acceptance

- support for research data, including large and complex data types

- gold Open Access which fosters wider collaboration and increased citations

- maximum visibility for your research: over $100 \mathrm{M}$ website views per year

At BMC, research is always in progress.

Learn more biomedcentral.com/submissions 